\title{
Detección de Perturbaciones en Soldadura Basado en el Monitoreo de la Acústica Aerotransportada del Proceso MIG / MAG
}

\author{
(Weld Interferences Detection Based in Airborne Acoustical Monitoring of MIG / MAG Process)
}

\author{
Eber Huanca Cayo $^{1}$, Sadek Crisóstomo Absi Alfaro ${ }^{1}$ \\ ${ }^{1}$ Universidade de Brasília,Departamento de Engenharia Mecânica,Brasília,Distrito Federal,Brasil,eber@unb.brsadek@unb.br
}

\begin{abstract}
Resumen
El sonido del proceso de soldadura es una consecuencia de la modulación en amplitud de la corriente por la tensión del arco. Experimentaciones pasadas demostraron que un soldador experimentado tiene una dependencia absoluta de la acústica en el control del proceso de soldadura. En este trabajo se presenta una nueva técnica de detección de defectos de soldadura basada en la estabilidad de la acústica aerotransportada del proceso MIG/MAG para el modo de transferencia por corto-circuito. Se determinaron parámetros estadísticos de la presión acústica generada por el arco eléctrico del proceso de soldadura MIG/MAG en el modo de transferencia por corto-circuito a partir de múltiples soldaduras en posición plana sobre cuerpos de ensayo libres de defectos. Con estos resultados se desarrollo un algoritmo de detección de defectos basado en una ventana móvil que se desplaza a través de las señales estadísticas calculadas a partir de la acústica del proceso. Finalmente sobre un conjunto de cuerpos de prueba con y sin defectos fueron hechas soldaduras en posición plana para la evaluación del desempeño de la técnica de detección de defectos propuesta. El algoritmo de detección de defectos basado en la acústica aerotransportada del proceso, mostró resultados satisfactorios.
\end{abstract}

Palabras clave: $M I G / M A G$; estabilidad; acústica; perturbación; defecto.

Abstract: The welding process's sound is a consequence of the modulation in amplitude of the current for the voltage of the electric arc. Previous experiments demonstrated that an experienced welder has an absolute dependence of the acoustics in the control of the welding process. In this work a new technique of detection of welding defects is presented based on the stability of the airborne acoustics of the process MIG/MAG for the transfer for short circuit mode. Statistical parameters of the acoustic pressure were determined and of the level of acoustic pressure for welding without defects from multiple tests; with these results have been development an algorithm of defects detection based on a mobile window that moves through the statistical signals calculated from the acoustics process. Finally a group of test plates within and without defects were tested; the algorithm based on the airborne acoustics of the developed process, showed satisfactory results for the detection of defects.

Key-words: $M I G / M A G$; stability; acoustical; perturbation; defect.

\section{Introducción}

Uno de los procesos de soldadura que actualmente es utilizado en la industria de la construcción metálica es la soldadura de arco con protección gaseosa, este proceso de soldadura también es conocido como MIG/MAG. Sus principales ventajas son la alta taza de transferencia metálica, elevada penetración y facilidad de soldar en todas las posiciones. Cuando el uso de este proceso de soldadura creció a escalas industriales, las exigencias y requerimientos de calidad de la soldadura en el mercado también fueron multiplicándose. La calidad de la soldadura actualmente es tema de múltiples investigaciones y discusiones, ya que su calificación envuelve diversos criterios de carácter subjetivo y que deben cumplir con las exigencias geométricas y estructurales suficientes para ser catalogadas como de buena calidad. Para logar estos requerimientos, se tiene que mantener una continuidad en

(Recebido em 25/11/2008; Texto Final em 01/06/2009). la estabilidad del proceso; esto sucede cuando el flujo de calor y masa de la punta del electrodo consumible hasta la poza de fusión a través del arco mantiene uniformidad en la transferencia; eventuales discontinuidades y/o trastornos en la transferencia podrían originar perturbaciones en la soldadura. Smith [1] y Adolfson [2] determinaron que la máxima estabilidad de un proceso de soldadura MIG/MAG en el modo de transferencia por corto-circuito, se produce cuando se tiene un máximo número de corto-circuitos en la transferencia, la desviación estándar de los periodos de corto-circuitos es mínima e se produce un mínimo nivel de salpicaduras. El arco eléctrico del proceso de soldadura, genera entre otros fenómenos físicos, la emisión de luminosidad, radiación infrarroja campos electromagnéticos, presión acústica. Es conocido que los soldadores especializados usan una combinación de información visual y acústica para el monitoreo y control del proceso de soldadura [3]. En el final de la década de los años 70's se logro medir indirectamente el voltaje del arco eléctrico a partir del sonido que éste produce [4], [5]; en ese trabajo se destacó la potencialidad de este 
método para el monitoreo de procesos de soldadura. Hasta el momento fueron propuestos algunos sistemas experimentales de monitoreo acústico para el proceso MIG/MAG pero éstos aun no fueron implementados comercialmente [6 - 15]. El monitoreo de la tensión de arco y corriente de soldadura permite la detección de perturbaciones del arco durante la soldadura y dependiendo de la naturaleza de éstas podrían interpretarse como defectos en las uniones soldadas. La detección y localización de defectos originados por perturbaciones del arco eléctrico durante la soldadura contribuye a reducir la rigurosidad de las pruebas de control de calidad tales como inspecciones visuales y/o ensayos no destructivos. La mayor parte de los equipos comerciales de monitoreo de tensión de arco y corriente utilizan sensores basados en divisores de tensión y efecto hall respectivamente y son instalados directamente en el proceso, esto quiere decir que existe conexión eléctrica entre los sensores y el proceso de soldadura. La conexión directa de sensores en el proceso de soldadura presenta dos desventajas considerables; por un lado el proceso de soldadura debido a su sensibilidad ante la variación de parámetros puede verse interferida por la presencia de sensores que tengan conexión eléctrica alterando la impedancia del arco eléctrico cuya variación podría generar inestabilidades que son indeseables y por otro lado las interferencias electromagnéticas que produce el arco eléctrico altera considerablemente la lectura realizada por los sensores que tienen conexión eléctrica con el proceso. El sonido del arco eléctrico representa el comportamiento de los parámetros eléctricos del arco, esto posibilita monitorear la estabilidad del proceso de soldadura a partir del sonido producido por el arco. El sonido producido por el arco eléctrico en el proceso de soldadura MIG/MAG puede representar el comportamiento de la secuencia de cortocircuitos e igniciones de la tensión de arco y por tanto abre la posibilidad de detectar acústicamente perturbaciones en el arco de soldadura [15]. La principal ventaja del monitoreo del sonido producido por el arco eléctrico radica en que no necesita tener conexiones eléctricas al proceso de soldadura, ya que el sonido es transmitido desde el arco eléctrico hasta el sensor acústico mecánicamente. Esto facilita la instalación del sensor en el proceso lo que reduce la posibilidad de alterar los parámetros eléctricos en el proceso de soldadura y al mismo tiempo su no conectividad eléctrica al proceso reduce la influencia de las interferencias electromagnéticas en el sensor acústico. En el presente trabajo se desarrolló una técnica de detección de defectos en soldadura basado en la evaluación de la estabilidad del proceso de soldadura utilizando información proveniente del sonido producido por el arco eléctrico.

La señal del sonido pasa por una etapa de pre-procesamiento para reducir el nivel de ruido ambiental luego de ello es procesada para obtener información del arco, estas operaciones implican en un elevado costo computacional, requiriendo sistemas computacionales de alto desempeño.

La relación entre la tensión y corriente del arco es representada por la ecuación (1) la cual da origen al sonido del arco eléctrico [4], [5]. En 1979 y 1980, Arata [16, 17] hizo mediciones confirmando la estrecha relación entre el nivel de presión acústica (que en adelante será citado como SPL del ingles Sound Pressure Level) y los parámetros eléctricos del arco, él también revelo la influencia del sonido en la poza de fusión. Además de ello descubrió que hay sincronización entre los impulsos acústicos y los corto-circuitos de la transferencia. Existe una sincronización junto con un retardo producido por la naturaleza aerotransportada del sonido [7, 18]. El SPL representa el comportamiento de la amplitud de la presión acústica para un intervalo $\Delta t$. El SPL en función de la presión acústica esta representada por la ecuación (2).

$$
\begin{aligned}
& S_{a}(t)=\frac{d(k \cdot V(t) \cdot I(t))}{d t} \\
& S P L=20 \cdot \log \left[\sqrt{\frac{1}{\Delta t} \int_{t_{0}}^{i_{0}+\Delta t}\left(\frac{S_{a}(t)}{S_{m}}\right)^{2} d t / p_{o}}\right]
\end{aligned}
$$

Donde: $S a(t) \quad$ - Señal de sonido $(\mathrm{V})$,

$\mathrm{Sm}$ - Sensibilidad del Micrófono (50 x 10-3 mV/Pa)

$V(t)$ - Tensión del arco (V)

$I(t)$ - Corriente eléctrica del arco (A)

$k$ - Factor geométrico

$S P L$ - Nivel de Presión Acústica (dB)

$P o$ - Referencia estándar de presión acústica $(20 \mu \mathrm{Pa})$

to - Tiempo de inicio de integración (s)

$\Delta t$ - Intervalo de tiempo de integración (s)

\section{Procedimiento Experimental}

\subsection{Instrumentos y Materiales}

Durante los experimentos realizados en el presente trabajo se utilizó una fuente de energía universal; adquirieron señales de tensión de arco y presión acústica utilizando un sistema divisor de tensión de arco y un decibelímetro B\&K - Type 2250 respectivamente. Ambas señales fueron muestreadas con una frecuencia de $20 \mathrm{kHz}$ utilizando la tarjeta de adquisición de datos PCI Eagle 703S que es controlado por el software de instrumentación virtual LabVIEW 8.2. En la Figura 1 (a) se muestra la distribución de los equipos utilizados. Estudios en psico-acústica determinaron que mientras la señal de sonido del proceso MIG/MAG no supere los $400 \mathrm{~ms}$ de retardo, éste será un buen indicador del comportamiento del proceso [6]. En diversos trabajos de monitoreo acústico de soldadura, cada autor instaló el micrófono a diversas distancias de la poza de fusión; $85 \mathrm{~mm}$ [5], $200 \mathrm{~mm}$ [18], $35 \mathrm{~mm}$ [11]. En este trabajo el micrófono se ubico a $150 \mathrm{~mm}$ de la poza de fusión y un ángulo de inclinación de $45^{\circ}$. (ver Fig. 1-b), para esta localización se tomó en cuenta la protección del micrófono contra las salpicaduras de la soldadura y la radiación térmica excesiva que pueda alterar la estabilidad, repetitividad y tiempo de respuesta del micrófono.

Los ensayos fueron realizados con alambre electrodo AWS A5.18 ER70S-6 de $1 \mathrm{~mm}$ de diámetro. Los cuerpos de ensayos fueron chapas de acero AISI 1020 de $200 \mathrm{~mm}$ x $50 \mathrm{~mm}$ x $6.50 \mathrm{~mm}$. El gas de protección usado fue la mezcla de argon y anhídrido carbónico M21 (ATAL 5A/Ar 82\% + CO2 18\%).

\subsection{Conjuntos de Soldaduras}

En la tabla 1 se muestran los parámetros de soldadura 


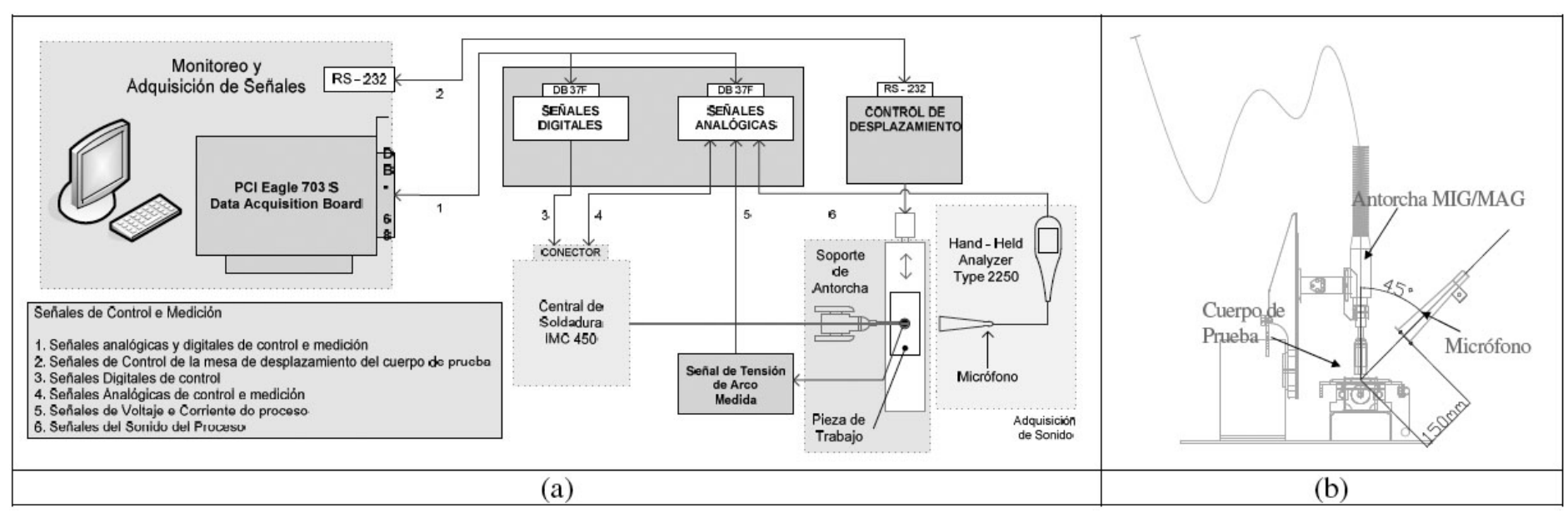

Figura 1. Hardware y Equipos usados en la Experimentación.

seleccionados. Estos parámetros fueron elegidos a partir de múltiples soldaduras siendo estos los que permiten realizar soldaduras con menor nivel de salpicaduras y mas uniformidad geométrica.

Tabla 1. Parámetros de Soldadura Seleccionados.

\begin{tabular}{c|c|c|c|c}
\hline Tensión & $\begin{array}{c}\text { WFS } \\
(\mathrm{m} / \mathrm{min})\end{array}$ & $\begin{array}{c}\text { ST. } \\
(\mathrm{mm} / \mathrm{s})\end{array}$ & $\begin{array}{c}\text { CTWD } \\
(\mathrm{mm})\end{array}$ & $\begin{array}{c}\text { Flujo de Gas } \\
(1 / \mathrm{min})\end{array}$ \\
\hline 20,0 & 6 & 10 & 12 & 15 \\
\hline
\end{tabular}

$C T W D=$ Distancia entre el contacto del electrodo y el cuerpo de ensayo (Contact Tip to Work Distance)

WFS =velocidad de alimentación del electrodo (Wire Feed Speed)

$S T=$ velocidad redesplazamiento de la antorcha de soldadura (Speed Travel)

En el presente trabajo se realizaron cuatro conjuntos de soldaduras (ver tabla 2) con los parámetros elegidos y ajustados en el equipo de soldadura. Se depositaron soldaduras en posición plana sobre los cuerpos de prueba siguiendo una trayectoria rectilínea de $180 \mathrm{~mm}$ como se muestran en la Figura 2. El primer conjunto consiste en soldaduras sin la presencia de perturbaciones, es decir sin la presencia de discontinuidades dimensiónales, estructurales ni de propiedades inadecuadas y cuya presencia es interpretada como un defecto [19]. Los tres siguientes conjuntos consisten en soldaduras con inducción de perturbaciones en la región central de la trayectoria de soldadura denominada como región de perturbación (ver figura 2). Las perturbaciones presentes en la región de perturbación para cada conjunto de soldaduras son: variación de Stand off (también conocido como CTWD), presencia de suciedad y ausencia de gas respectivamente, estas perturbaciones generan discontinuidades estructurales tales como las irregularidades en los cordones de soldadura, falta de fusiona si como la presencia de porosidades.

Tabla 2. Conjuntos de Soldaduras y Perturbaciones Inducidas.

\begin{tabular}{c|l|c|c}
\hline \multicolumn{2}{c|}{ Conjunto de Soldadura } & No. de Soldaduras & Perturbación Inducida \\
\hline 1 & Libre de Perturbaciones & 20 & Sin perturbaciones \\
\hline 2 & Variación de CTWD & 10 & Variación de CTWD de $12 \mathrm{~mm}$ a $10 \mathrm{~mm}$ \\
\hline 3 & Presencia de Suciedad & 10 & Presencia de grasa en la Región de Perturbación \\
\hline 4 & Ausencia de Gas & 10 & Ausencia de gas en la Región de Perturbación \\
\hline
\end{tabular}

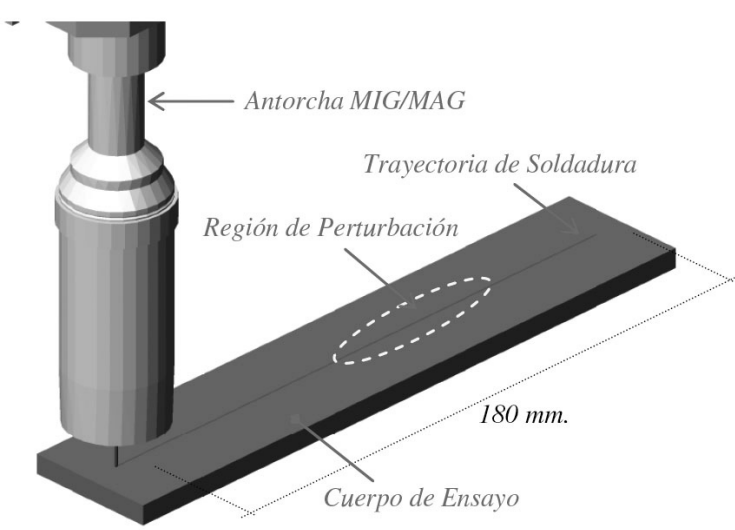

Figura 2. Posición de Soldadura y Localización de Perturbaciones.
La perturbación inducida por variación de CTWD se hizo colocando una chapa con tres agujeros en su superficie (ver Figura 7-a); las dimensiones de la chapa son de $2 \mathrm{~mm}$ de espesor, 20 $\mathrm{mm}$ de ancho y $50 \mathrm{~mm}$ de largo. Esta chapa fue colocada sobre la región de perturbación. La presencia de ésta perturbación genera discontinuidades estructurales debido a la irregularidad de los cordones de soldadura; la variación del Stand Off da como origen a la variación de la longitud del arco eléctrico lo que produce inestabilidades en las igniciones del arco generándose así discontinuidades estructurales; estas inestabilidades únicamente se manifiestan cuando la soldadura pasa por el inicio, final y los agujeros de la chapa. La perturbación inducida por presencia de suciedad se realizó colocando grasa sobre la región de perturbación. Durante el paso de la soldadura por la región de perturbación, se produjo inestabilidades en las igniciones del arco 


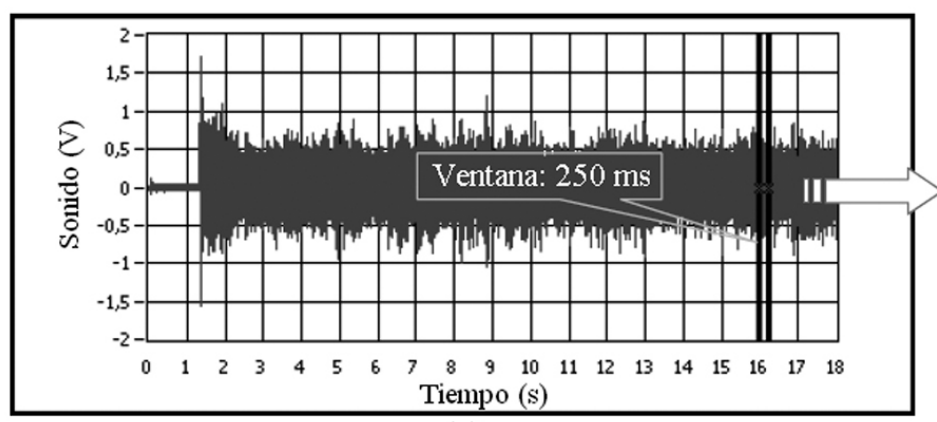

(a)

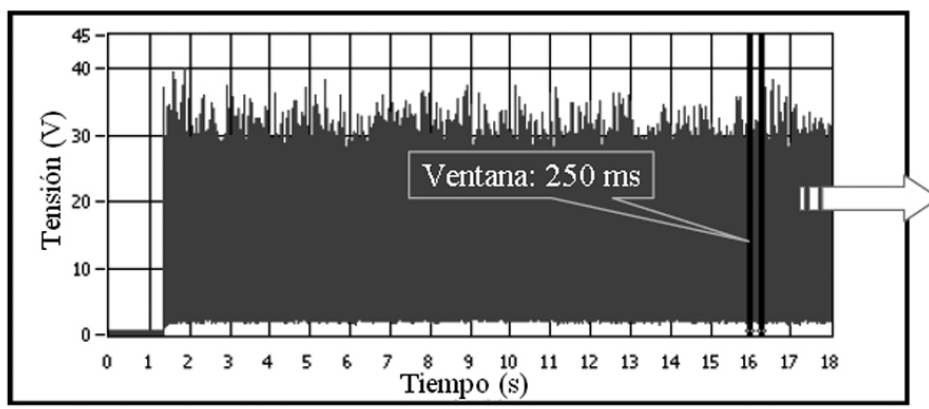

(b)

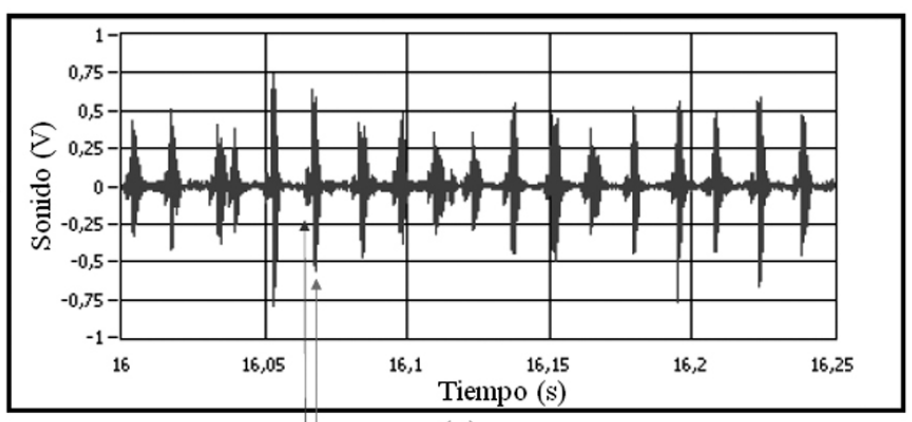

(c)

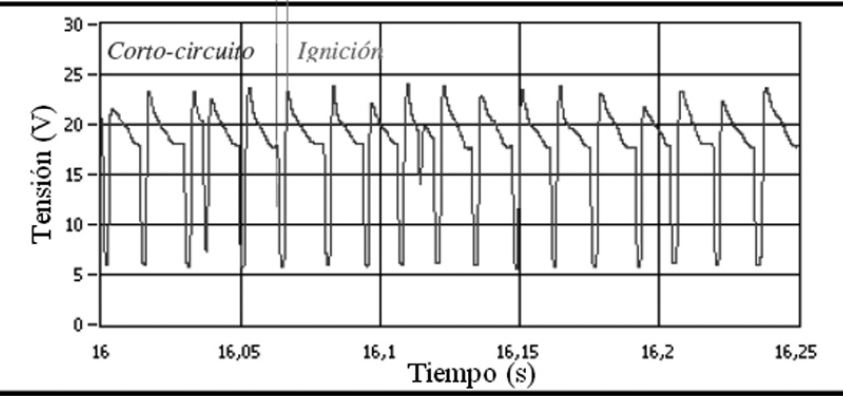

(d)

Figura 3. Señales de Presión Acústica y Tensión de Arco en Proceso MIG/MAG.

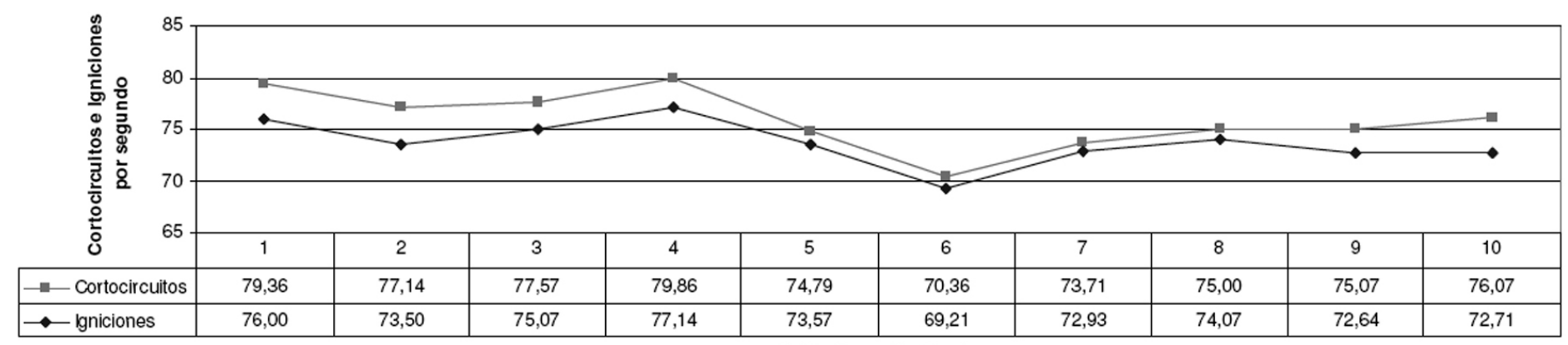

Soldaduras Reallzadas

Figura 4. Corto-circuito e Igniciones de Arco para Condiciones de Máxima Estabilidad.

originándose trastornos dramáticos en los ciclos de trasferencia metálica que como consecuencia se formaron discontinuidades estructurales de soldadura en la trayectoria seguida. La perturbación inducida por la ausencia de gas de protección se realizó interrumpiendo el paso de éste cerrando la válvula de suministro de gas durante el paso por la región de perturbación. La ausencia de gas genero discontinuidades estructurales debido a la presencia de porosidades sobre la soldadura así como la elevación del nivel de salpicaduras debido a la contaminación de la atmosfera cuando se produce la ignición del arco así como la transferencia metálica desde el electrodo hasta la poza de fusión. En cada unos de los experimentos de cada conjunto de soldaduras se adquirieron señales de tensión y presión acústica del arco eléctrico.

\section{Resultados y Discusión}

\subsection{Señal Eléctrica y Acústica en el Proceso de Soldadura MIG/MAG}

El modo de transferencia por corto-circuito en el proceso de soldadura se produce cuando se tiene una secuencia de cortocircuitos e igniciones del arco de soldadura. Estos fenómenos se ven reflejados en la acústica del arco expresada por la ecuación 1 y 2 . Debido a esto es posible calcular el numero de cortocircuitos e igniciones del arco a partir de la acústica del arco [13, 14]. En la Figura 3(a) y (b) se presentan las señales de presión de sonido y tensión de arco respectivamente. En las Figuras 3(c) y (d) se presenta una ventana móvil de $250 \mathrm{~ms}$. de las señales descritas anteriormente, en ellas es posible notar como se manifiesta en el sonido los corto-circuitos e igniciones del arco eléctrico. Un corto-circuito en el arco se reproduce en el sonido como un pulso de pequeña amplitud, mientras que una ignición se preproduce en el sonido como un impulso de gran amplitud. Debido al ruido ambiental a veces es poco notorio el impulso acústico que reproduce el corto-circuito. Sin embargo el cortocircuito y la ignición del arco son dos fenómenos que siempre ocurrirán uno después del otro, por tanto es posible medir la frecuencia de igniciones y la periodicidad de estos a partir de los 
impulsos acústicos producidos por el arco eléctrico.

En la Figura 4 podemos notar el numero de corto-circuitos de tensión de arco y el numero de igniciones de arco promedio para 10 soldaduras del primer grupo de soldaduras (soldaduras sin presencia de discontinuidades). En ella podemos notar la estrecha semejanza de los resultados medidos destacando que fueron obtenidos por métodos diferentes, uno eléctrico y otro acústico; esta similitud entre ambos resultados confirma que el sonido del arco puede representar el comportamiento de la transferencia metálica del proceso de soldadura MIG/MAG en el modo de transferencia por corto-circuito.

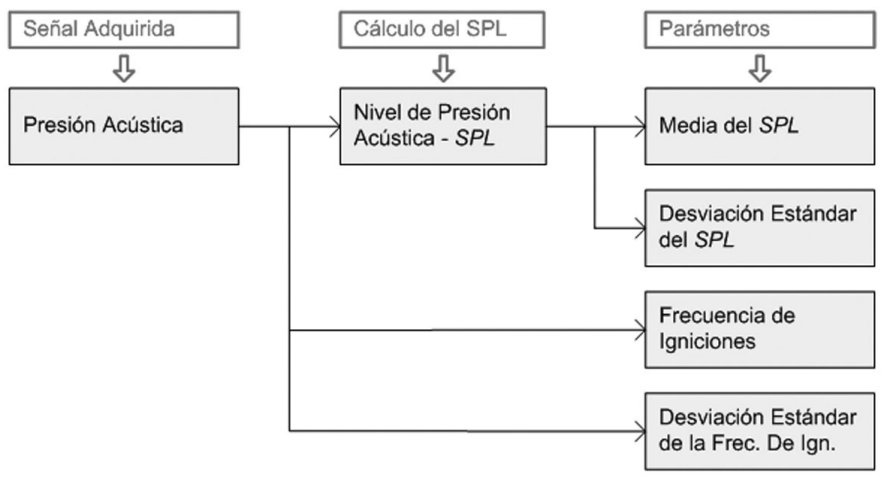

Figura 5. Parámetros de Estabilidad Acústica Calculados.

\subsection{Parámetros de Estabilidad Acústica}

En laFigura 5 semuestran los cuatros parámetros de estabilidad acústica establecidos que son: la media del SPL, desviación estándar del SPL, la frecuencia de igniciones y la desviación estándar de la frecuencia de igniciones. Los dos primeros parámetros son calculados a partir del SPL calculado mediante la ecuación 2 y los dos últimos parámetros son calculados a partir de la señal de presión acústica. Para la evaluación de estos parámetros de estabilidad acústica fue necesario determinar una ventana móvil de mínima duración de tiempo que contenga la cantidad suficiente de datos de tal forma que con éstos se pueda representar el comportamiento estadístico de cada parámetro de estabilidad acústica. Puesto que el comportamiento de la amplitud de la señal de presión acústica guarda una estrecha relación con el comportamiento de la transferencia metálica que es de naturaleza estocástica, inferimos que la actividad acústica del arco eléctrico también tiene un comportamiento de naturaleza estocástica. Experimentaciones anteriores representaron el comportamiento estadístico de señales estocásticas del proceso MIG/MAG con ventanas móviles de datos de $150 \mathrm{~ms}$ [15] y $200 \mathrm{~ms}$ [20]. Cuando mayor sea la duración de la ventana móvil de datos, mayor será la posibilidad de representar las características estadísticas de la señal estocástica, sin embargo el incremento de la duración de la ventana móvil de datos también reduce la resolución del parámetro calculado. Bajo este criterio los parámetros calculados: media del SPL y las desviaciones estándar del SPL así como de la frecuencia de igniciones acústicas que están en función de tiempo, son calculados a partir de las ecuaciones 3 y 4 utilizando ventanas móviles de datos de $250 \mathrm{~ms}$.

\subsection{Detección de Perturbaciones}

Cada perturbación inducida generó discontinuidades estructurales sobre la soldadura. De acuerdo con las exigencias de calidad para la soldadura, una discontinuidad puede ser considerada como perjudicial para su utilización futura, convirtiéndose, de esta manera, en un defecto y exigiendo acciones correctivas [19]. En el presente trabajo se detectaron la presencia de perturbaciones inducidas que generaron discontinuidades. Para ello se utilizó una ventana móvil de comparación de datos de $250 \mathrm{~ms}$ de duración que fue haciendo el barrido a lo largo de cada parámetro acústico previamente calculados dentro de la región de análisis que corresponde a las señales adquiridas a partir del tercer segundo hasta el segundo 17, región donde se garantiza que las señales de tensión y corriente alcanzaron un nivel de estacionario (ver Figura 6 (a)). Como resultado de un análisis estadístico de los parámetros acústicos del primer conjunto de experimentos en soldadura (sin la presencia de perturbaciones que generen discontinuidades) se establecieron los niveles aceptables máximos y mínimos de variación de estabilidad (ver tabla 3). El rango entre los valores mínimos y máximos es también llamado rango de estabilidad. En la Figura 6(a) se muestra una soldadura sin la presencia de perturbaciones; en las Figuras 6 (b-e), se muestra el comportamiento del SPL, el desvío estándar del SPL, el numero de igniciones y la desviación estándar de los periodos de igniciones por cada ventana móvil.

Tabla 3. Niveles Máximos y Mínimo Establecidos.

\begin{tabular}{l|c|c|c|c}
\hline Niveles Establecidos & Media del SPL $(\mathrm{dB})$ & $\begin{array}{c}\text { Desviación Estándar } \\
\text { del SPL }(\mathrm{dB})\end{array}$ & $\begin{array}{c}\text { Frecuencia de Igniciones } \\
\text { (ign./ventana) }\end{array}$ & $\begin{array}{c}\text { Desviación Estándar del } \\
\text { período de igniciones }\end{array}$ \\
\hline Máximo $(\mathrm{Mx})$ & 84,00 & 4,00 & 14,00 & 0,0073 \\
\hline Mínimo $(\mathrm{Mn})$ & 78,50 & 1,00 & 7,50 & 0,0025 \\
\hline
\end{tabular}

La ecuación (5) es una función binaria que representa la ventana móvil de comparación de parámetros. Esta función binaria compara cada parámetro acústico en función del tiempo con los respectivos niveles máximos y mínimos establecidos en la tabla 3. Dicha función binaria toma el valor de 1 (uno) cuando una muestra del parámetro evaluado en función del tiempo supera el limite admisible superior o inferior correspondiente y 0 (cero) cuando se encuentra dentro de rango admisible. La detección de variaciones de los parámetros fuera del rango de estabilidad realizada mediante la ecuación (5) para cada parámetro es interpretado como la presencia de alguna perturbación que posiblemente origine una inestabilidad en la soldadura que represente un defecto. 


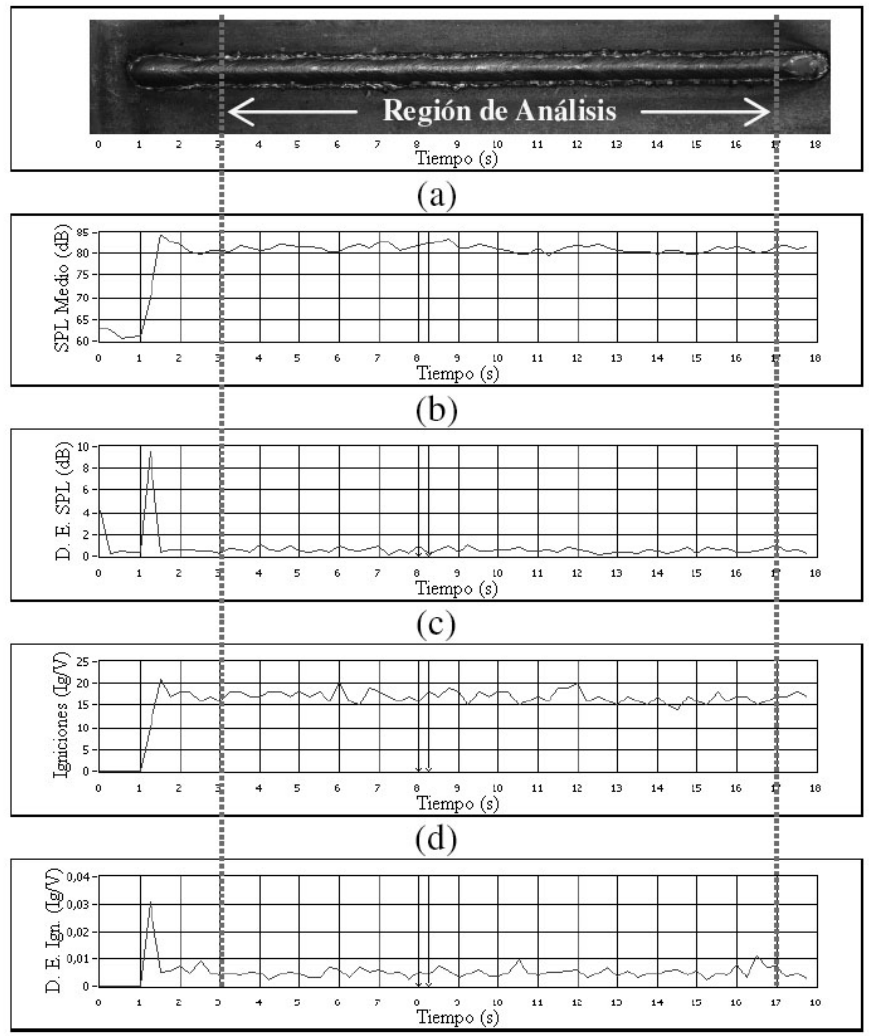

(e)

Figura 6. Soldadura Libre de Perturbaciones.

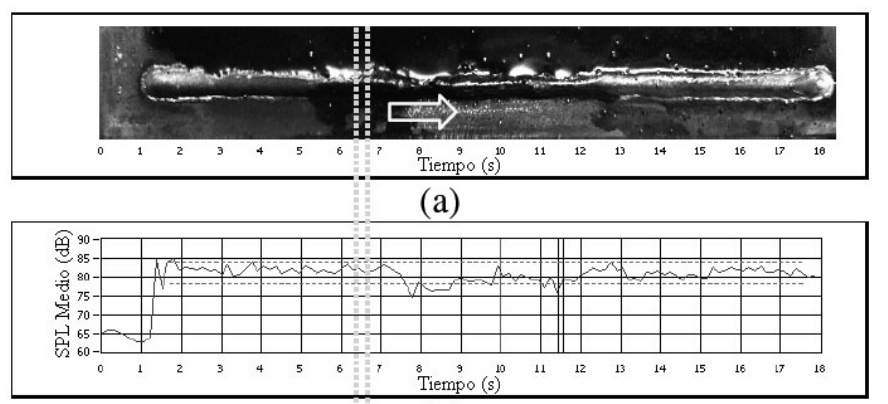

(b)

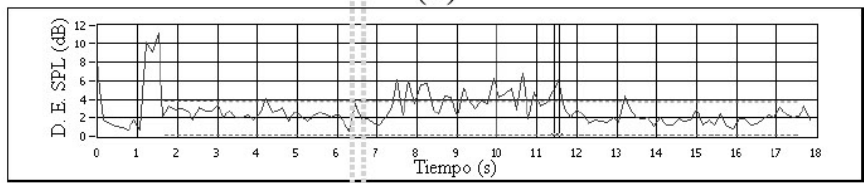

(c)

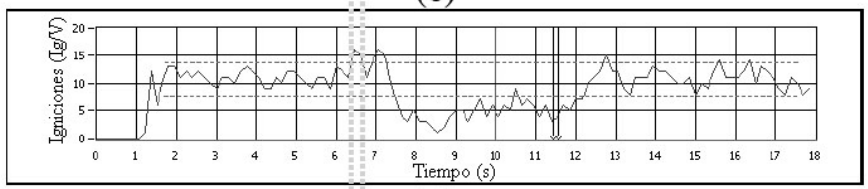

(d)

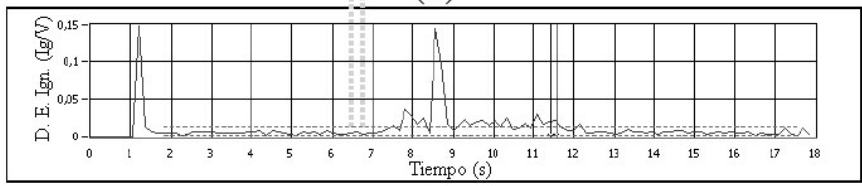

(e)

Figura 8. Soldadura con Perturbación por Presencia de Suciedad en la Trayectoria.

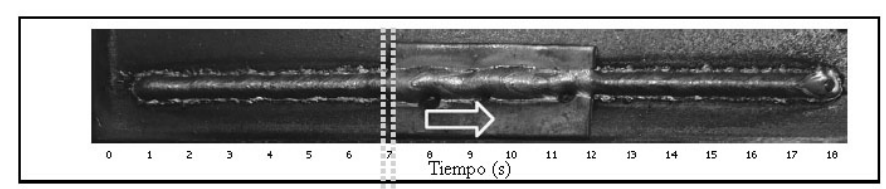

(a)

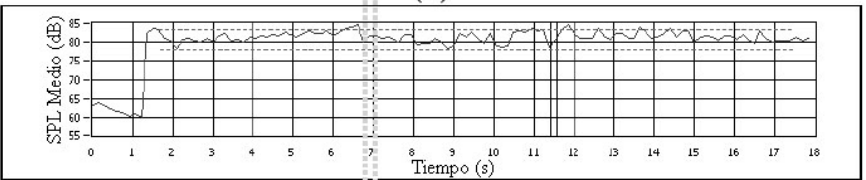

(b)

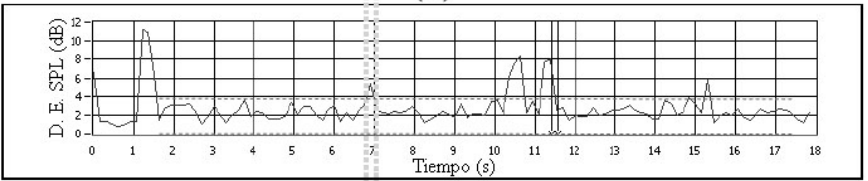

(c)

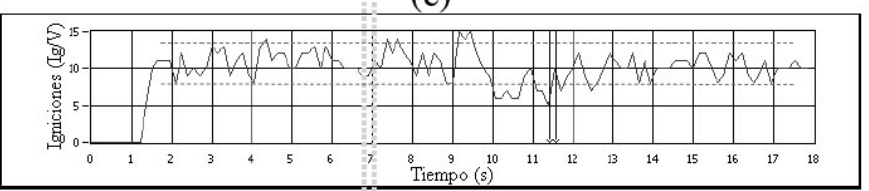

(d)

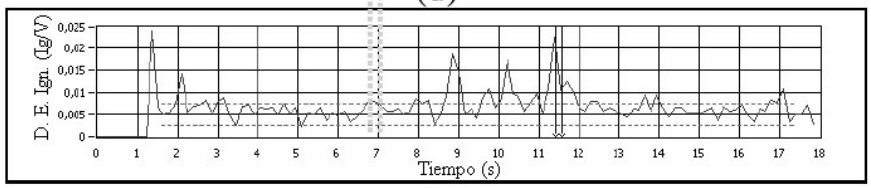

(e)

Figura 7. Soldadura con Perturbación por Variación de CTWD.

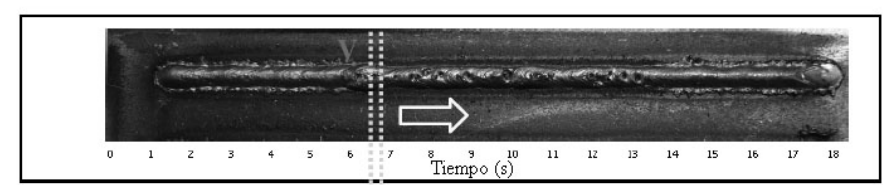

(a)

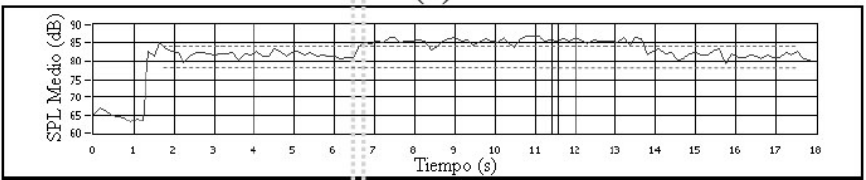

(b)

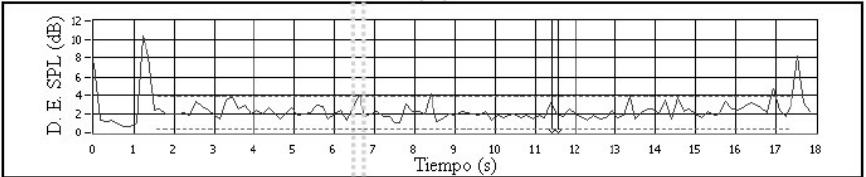

(c)

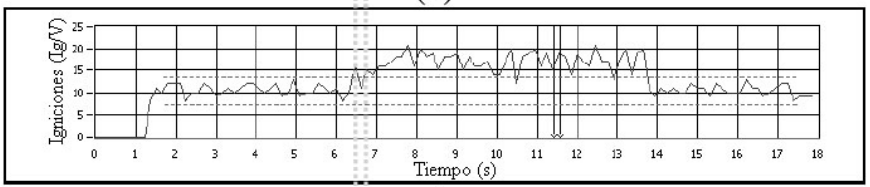

(d)

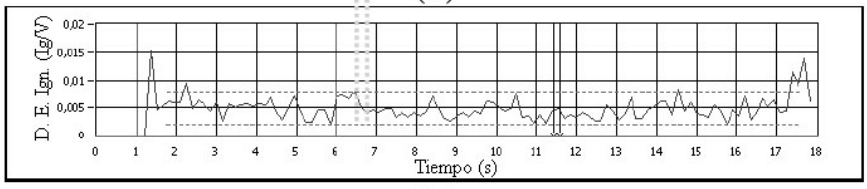

(e)

Figura 9. Soldadura con Perturbación por Ausencia de Gas de Protección. 


$$
\begin{gathered}
\overline{x_{i}}=\frac{1}{n} \sum_{j=1}^{n} x_{i}=\frac{1}{n}\left(x_{1}+\ldots+x_{n}\right) \\
S_{i}=\sqrt{\frac{1}{n} \sum_{j=1}^{n}\left(x_{j}-\overline{x_{i}}\right)^{2}} \\
W_{i}=\left\{\begin{array}{ccc}
1, & \text { si } & \left(V_{i=1}^{k}>M x\right) \text { or } \quad\left(V_{i=1}^{k}<M n\right) \\
0, & \text { si } & \left(M n \leq V_{i=1}^{k} \leq M x\right)
\end{array}\right.
\end{gathered}
$$

Donde: $\quad \bar{x}_{i}: \quad$-Media Aritmética de la i-esima ventana móvil de análisis

$S_{i}$ : -Desviación Estándar de la i-ésima ventana móvil de análisis

$n$ : $\quad$-Numero de Datos Componentes de la ventana móvil de análisis móvil de análisis

$$
x_{j}: \text {-j-esimo Dato Componente de la ventana }
$$

$W_{i}: \quad$-Test i de comparación

$V_{i}^{k}$ : -i-esima ventana móvil de dato medio comparado con la referencia $\mathrm{k}$ establecido

Mx: -Máximo valor de referencia de estabilidad establecido

Mn: -Mínimo valor de referencia de estabilidad

\subsection{Desempeño de la Técnica de Detección de Perturbaciones}

En las Figuras 7, 8 y 9, se muestran ensayos de simulación de defectos por variación de stand off, presencia de grasa sobre los metales y ausencia de gas respectivamente. En las Figuras 7(b - e), 8(b - e) y 9(b - e) se muestran los parámetros de estabilidad acústica resultantes para cada grupo de soldaduras respectivamente; estos parámetros son: SPL medio, desviación estándar de SPL, frecuencia de Igniciones y desviación estándar de la frecuencia de igniciones. En estas Figuras también podemos la presencia de los niveles máximos y mínimos establecidos para cada señal (línea punteada) así como la ventana móvil V. Esta ventana de análisis se desplaza a lo largo de las señales calculada a partir de la acústica del arco eléctrico detectando defectos a partir de las ecuaciones (5) y (6).

En las Figura 8(d) y 9(d) (frecuencia de igniciones) y en las Figuras 7(c) y (e) (desviaciones estándar de SPL y frecuencia de igniciones) podemos observar como se comportan los parámetros de estabilidad ante la presencia de perturbaciones en la trayectoria de las soldaduras. Estas variaciones atípicas de los parámetros de estabilidad acústica permiten detectar las perturbaciones que originan inestabilidades en arco.

Para la validación de la nueva técnica de detección acústica de perturbaciones en soldaduras, se realizaron 10 (diez) soldaduras de cada conjunto de soldaduras. Estas soldadura fueron realizadas con los parámetros establecidos en la tabla 1. En estas soldaduras finales se pudieron detectar la totalidad de las soldaduras defectuosas originadas por la presencia de grasa y ausencia de gas. En los defectos generados por la variación de stand off, solo pudieron ser detectadas 5 ensayos como defectuosos. Dentro de los 10 ensayos correspondientes a soldaduras sin presencia de defectos, todas fueron evaluadas correctamente. De esta manera mostramos que es posible detectar discontinuidades estructurales como la irregularidad en los cordones de soldadura, la falta de fusiona si como la presencia de porosidades, defectos que pueden ser inspeccionados visualmente. Un análisis detallado y minucioso del comportamiento de los parámetros de estabilidad acústica ante la presencia de perturbaciones permitiría la caracterización de cada tipo de perturbación abriendo la posibilidad de poder identificar el tipo de defecto en la soldadura.

\section{Conclusiones}

El uso de la acústica del arco de soldadura, es una herramienta factible para la evaluación de la estabilidad del proceso MIG/ MAG; desde el hecho que las inestabilidades del proceso se ven reflejadas en la presión acústica emitida.

El algoritmo desarrollado, presenta mejores resultaron ante inestabilidades producidas por defectos originados por la ausencia de gas y presencia de suciedad sobre los cuerpos de prueba en comparación a las inestabilidades originadas por la variación del stand Off.

Las inestabilidades generadas por la variación de stand off no presentan grandes variaciones o por lo menos variaciones perceptibles en el SPL y la frecuencia de igniciones, esto se debe a que la inestabilidad se produce solo en el momento de la variación del stand off, luego de ello el arco eléctrico de estabiliza para el nuevo stand off y por tanto las emisiones acústicas también se estabilizan. Sin embargo este tipo de defectos presentan variaciones notorias en la desviación estándar del SPL y la periodicidad de igniciones únicamente durante la variación del stand off. Los defectos originados por la presencia de grasa se caracterizan por la abrupta reducción de la frecuencia de igniciones así como la desviación estándar de la periodicidad de igniciones. Los defectos originados por la ausencia de gas de protección se reflejan en el incremento de la frecuencia de igniciones, no obstante ello los demás parámetros permanecen estables.

\section{Agradecimientos}

Los autores agradecen al CNPq, FINATEC y al Profesor P. J. Modenesi por los comentarios al trabajo.

\section{Referencias Bibliográficas}

[1] SMITH, A. A., Characteristics of the short - circuiting CO2 - Shielded Arc, Proceedings, Institute of Welding Symposium on Physics of the Welding Arc, London, 1962, p75 - 91.

[2] ADOLFSON, S. et al, On-line quality monitoring in shortcircuit gas metal arc welding, Welding Journal, p. no2 59s-73s 
February, 1999.

[3] KRALJ, V., Biocybernetic investigations of hand movements of human operator in hand welding. IIW/IIS Doc. 212-140-68, 1968.

[4] DROUET, MICHEL G., NADEAU, F., Pressure Waves due to Arcing Faults in a Substation, IEEE Transactions on Power Apparatus and Systems, Vol. No.5, 1979.

[5] DROUET, MICHEL G., NADEAU, F., Acoustic measurement of the arc voltage applicable to arc welding and arc furnaces, J. Phys. E: Sci. Instrum., Vol. 15, 1982.

[6] MANSOOR, A.M., HUISSOON, J.P., Acoustic Identification of the GMAW Process, 9th Intl. Conf. on Computer Technology in Welding, , pp312-323 Detroit, USA, Sept. 1999.

[7] TAM J., HUISSOON, J., Developing Psycho-Acoustic Experiments in Gas Metal Arc Welding, Proceedings of the IEEE, International Conference on Mechatronics \& Automation, Niagara Falls, Canada, July 2005.

[8] GRAD L., ET AL, Feasibility study of acoustic signals for on-line monitoring in short circuit gas metal arc welding, International Journal of Machine Tools and Manufacture Volume 44, Issue 5, Pages 555-561, April 2004.

[9] POOPAT, B., WARINSIRIRUK, E., Acoustic signal analysis for classification of transfer mode in GMAW by noncontact sensing technique, Songklanakarin Journal of Science and Technology, Vol. 28, Issue 4, pages/rec.No: 829840, University of Technology Thonburi, Thungkru, Bangmod, 2006, Bangkok, Thailand.

[10] CAYO EBER H., ABSI ALFARO, S.C., Medición de la Calidad en Soldadura Basado en Sensoreamiento Acústico, $8^{\circ}$ Congreso Iberoamericano de Ingeniería Mecánica, 2007, Cusco - Perú. CIBIM8. Lima - Perú : Pontificia Universidad Católica del Perú, 2007. v. 1. p. 1068-1079.

[11] ČUDINA, M., PREZELJ, J., POLOJNAR I., Use of Audible Sound For On-Line Monitoring of Gas Metal Arc Welding Process, Croatian Metallurgical Society, Metalurgija 47 (2008) 2, 81-85, 2008, Zagreb, Croatia.

[12] CAYO EBER H., ABSI ALFARO, S.C., Evaluación Acústica de la Estabilidad del Proceso de Soldadura MIG/MAG para el Modo de Transferencia Corto-circuito, $3^{\circ}$ Congreso Nacional de Ingenierías Mecánica, Eléctrica, Electrónica y Mecatrónica, Universidad Autónoma Metropolitana, 2008, Azcapotzalco-Ciudad de Mexico, Mexico, v. 3, s. 4, pp. 399405.

[13] CAYO EBER H., ABSI ALFARO, S.C., Weld Transference Modes Identification Through Sound Pressure Level in GMAW Process, Sixteenth International Scientific Conference on Achievements of Mechanical and Materials Engineering AMME'2008, June 2008, Gliwice, Poland, p. 1.15, p.73. ISBN 83-89728-47-8, EAN 9788389728470.

[14] CAYO, EBER H.; ABSI ALFARO, S.C., Welding Quality Measurement Based On Acoustic Sensing, COBEM2007, 2007, Brasília. 19th International Congress of Mechanical Engineering. São Paulo: ABCM, 2007. v. 1. p. 2200-2200-11.

[15] CAYO, EBER H., Monitoramento, Detecção E Localização De Defeitos Na Solda Baseada No Sensoriamento Da Pressão Acústica Do Arco Elétrico No Processo GMAW-S: 2008. 108P. Disertación (Maestría en Sistemas Mecatrónicos) - Facultad de
Mecánica, Universidad de Brasilia, Distrito Federal.

[16] ARATA, Y. et al. Investigation of Welding Arc Sound (Report 1) - Effect of Welding Method and Welding Condition on Welding Arc Sound. Reports Japan Welding Research Institute, 1979.

[17] ARATA, Y.etal. Investigation of Welding Arc Sound (Report 2) -Evaluation of Hearing Acuity and Some Characteristics of Sound. Reports Japan Welding Research Institute, 1980.

[18] WARINSIRIRUK E., POOPAT B., Investigation of Metal Transfer of ER70S-6 Filler metal in MAG-M Welding by Acoustic Signal Detection, The Fourth Thailand Materials Science and Technology Conference, Bangkok, Thailand, April 2006.

[19] VILlani, P., MODENESI, P. E BRACARENSE, A., (2005), Soldagem Fundamentos e Tecnologia, 1.ed. Belo Horizonte - Brasil, Editora UFMG 362 p.

[20] ABSI, S. C., CARVAlHO, G.C., DA CUNHA, F.R., (2006), A statistical approach for monitoring stochastic welding processes, Journal of Materials Processing Technology 175 $4-14$. 